OPEN ACCESS

Edited by:

Robert Vierkant,

Mayo Clinic, United States

Reviewed by:

Valerio Gallotta,

Catholic University of the Sacred

Heart, Italy

Vito Andrea Capozzi,

University Hospital of Parma, Italy

*Correspondence:

Zhengyu $L$

zhengyuli01@126.com

Specialty section:

This article was submitted to Gynecological Oncology,

a section of the journal

Frontiers in Oncology

Received: 30 September 2021 Accepted: 29 October 2021 Published: 16 November 2021

Citation:

Yang Y, Huang Y and Li Z (2021) The Surgeon's Proficiency Affected Survival Outcomes of Minimally Invasive Surgery for Early-Stage Cervical Cancer: A Retrospective Study of 851 Patients.

Front. Oncol. 11:787198. doi: 10.3389/fonc.2021.787198

\section{The Surgeon's Proficiency Affected Survival Outcomes of Minimally Invasive Surgery for Early-Stage Cervical Cancer: A Retrospective Study of 851 Patients}

\author{
Ying Yang ${ }^{1,2}$, Yue Huang ${ }^{1,2}$ and Zhengyu $\mathrm{Li}^{1,2 *}$ \\ 1 Department of Gynecology and Obstetrics, West China Second University Hospital, Sichuan University, Chengdu, China, \\ ${ }^{2}$ Key Laboratory of Pediatric Diseases and Birth Defects of Ministry of Education, West China Second University Hospital, \\ Sichuan University, Chengdu, China
}

Purpose: To compare the clinical outcomes of patients with early-stage cervical cancer who underwent minimally invasive surgery (MIS) by surgeons in different phases and evaluate whether the proficiency of surgeons affects the survival outcomes.

Materials and Methods: A total of 851 patients with early-stage cervical cancer who underwent radical hysterectomy between January 2008 and June 2018 (every year from January to June) at a tertiary hospital were retrospectively analyzed. We categorized patients into four phases according to their sequence (phase one, 1-10 cases; phase two: 11-20 cases; phase three: 21-30 cases; phase four: > 30 cases). Demographics and clinical and pathological data were collected and analyzed.

Results: There were no statistical differences between the open surgery and MIS groups regarding three- and five-year overall survival (OS) and disease-free survival (DFS). The OS and DFS of patients in the MIS group in phase one were significantly lower than those in later phases and those in the open surgery group after adjustment (OS, $P=0.009$; HR, 2.896; 95\%Cl, 1.303-6.435; DFS, $P=0.009$; HR, 2.712; 95\%Cl, 1.289-5.706). Survival outcomes were not statistically significant when comparing different surgeons.

Conclusion: The phase one cases of MIS had lower OS and DFS than those in later phases and those in the open surgery group. Thus, we suggest that the proficiency of surgeons is associated with survival outcomes of MIS. Favorable outcomes can be obtained after a certain number of MIS cases.

Keywords: early-stage cervical cancer, minimally invasive surgery, open surgery, surgeon's proficiency, survival outcomes 


\section{INTRODUCTION}

According to statistics from the International Agency for Research on Cancer, the incidence of cervical cancer was 604,000 in 2020, making it the fourth most common gynecological cancer worldwide (1). Radical hysterectomy $(\mathrm{RH})$ with lymphadenectomy remains one of the preferred treatments for patients with cervical cancer diagnosed at the International Federation of Gynecology and Obstetrics (FIGO) stage IA-IIA (2). In recent decades, minimally invasive surgery (MIS) has become more common, displacing the use of traditional open surgery for early-stage cancer. However, the Laparoscopic Approach to Cervical Cancer (LACC) trial observed that disease-free survival (DFS) at 4.5 years and overall survival (OS) at three years of MIS were significantly lower than those of open surgery among women with stage IA1IB1 cervical cancer, which remains controversial (3). Potential explanations include increasing tumor spillage due to the application of a uterine manipulator, the effect of $\mathrm{CO}_{2}$ insufflation, the volume of surgery, and the surgeon's proficiency (4-7). Several studies have focused on the correlation between surgeon proficiency and clinical outcomes in recent years. Lan Ying Li et al. found that compared to open surgery, more cases were required for surgeons performing minimally invasive $\mathrm{RH}$ to reach an acceptable five-year DFS (8). Kim et al. demonstrated that surgeons' proficiency in the MIS group significantly affected progression-free survival (PFS) (9). Liu et al. concluded that the learning curve could be a probable reason for poor outcomes of MIS by analyzing stage IB cervical cancer patients treated with RH by one surgeon for 15 years (10). Nevertheless, existing studies have limitations such as low sample sizes, incomplete follow-up information, and the absence of clinicopathological features of patients. There is still insufficient evidence regarding whether a surgeon's proficiency is associated with survival outcomes in MIS. We aimed to explore the effects of surgeons' proficiency in MIS on short- and longterm clinical outcomes and whether it accounts for the clinical outcomes of MIS.

\section{MATERIALS AND METHODS}

\section{Study Design and Participants}

This study was approved by the Institutional Ethics Committee of West China Second University Hospital, and all participants provided their written informed consent to participate in this study. Cervical cancer patients with consecutive FIGO (2009) stage IA-IIA treated with RH between January 2008 and June 2018 (every year from January to June) were retrospectively analyzed. The inclusion criteria were: (1) patients with FIGO stage IA1 with lymphovascular space invasion (LVSI), IA2, IB, and selected IIA cervical cancer; (2) patients who underwent standard surgical treatment, which was performed by five specific surgeons according to the National Comprehensive Cancer Network guidelines, a modified RH (Type B of the Querleu and Morrow (Q-M) surgical classification system) with pelvic lymphadenectomy (PLND) in stage IA1 with LVSI and stage IA2, and an RH (Type C of the Q-M surgical classification system) with PLND with/without para-aortic lymphadenectomy in stage IB to IIA $(2,11,12)$. (3) Patients with histological subtypes of squamous cell carcinoma, adenocarcinoma, or adenosquamous carcinoma, regardless of histological grading. The exclusion criteria were as follows: (1) patients with incomplete data or complete loss to follow-up; (2) patients with severe fundamental diseases such as immune deficiency or other malignant tumors; and (3) patients with cervical cancer in pregnancy.

Complete information, including demographics and clinical and pathological information, was extracted from the hospital information system by two investigators (Y.Y. and Y.H.). The demographics extracted included age, menstruation (menopause or not), and body mass index (BMI). The clinical information extracted included diagnosis, FIGO (2009) stage, surgical approach, surgeon name, date of surgery, hospital stay, duration of surgery, estimated blood loss, $\mathrm{CO}_{2}$ pneumoperitoneum in MIS, number of resected lymph nodes, pre- and post-operative treatment, and imaging data. Pathological information included histologic subtype, grading, LVSI, stromal invasion depth, parametrial involvement, vaginal margin involvement, and lymph node metastasis. Recurrence was confirmed by clinical findings, radiological examinations, and pathology reports. OS and DFS were the primary outcomes of this study. OS was defined as the time interval between the date of surgery and the date of death. DFS was defined as the time interval between the date of surgery and either the date of the first recurrence or death.

\section{Statistical Analysis}

The data were analyzed using the Statistical Package for the Social Sciences software version 22.0 (IBM Corp, Armonk, NY, USA). Missing values were statistically imputed by multiple imputations using logistic regression and predictive mean matching (13). The enumeration data were analyzed using the chi-square test. The measurement data were analyzed via t-test and Mann-Whitney $\mathrm{U}$ test between two groups, while an analysis of variance and Kruskal-Wallis $\mathrm{H}$ test were used to compare multiple groups. Survival curves were generated using the Kaplan-Meier method and analyzed using the log-rank test and multivariable Cox proportional hazards regression models. All the $P$-values reported are two-sided. A $P$-value of $<0.05$ was considered statistically significant.

\section{RESULTS}

\section{Study Population}

A total of 851 patients were included in the study: 510 in the open surgery group and 341 in the MIS group (no robotic RH). Their general characteristics are summarized in Table 1. The mean age of the patients was 46.51 years (range 18-73 years; standard deviation [SD] 9.33), and most patients (471, 55.35\%) were diagnosed with stage IB1. There were no significant differences between the open surgery and MIS groups in terms 
TABLE 1 | General characteristics of the study population stratified by surgical approach.

\begin{tabular}{|c|c|c|c|}
\hline Characteristic & Open surgery $(n=510, \%)$ & Minimally invasive surgery $(n=341, \%)$ & $P$ value \\
\hline Age (year) (mean $\pm \mathrm{SD}$ ) & $46.70 \pm 9.39$ & $46.22 \pm 9.25$ & 0.456 \\
\hline Menopause & 170 (33.3) & $114(33.4)$ & 0.976 \\
\hline $\mathrm{BMl}(\mathrm{kg} / \mathrm{m} 2)($ mean $\pm \mathrm{SD})$ & $22.58 \pm 2.80$ & $23.06 \pm 4.36$ & 0.074 \\
\hline Histologic subtype & & & 0.297 \\
\hline squamous-cell carcinoma & $417(81.8)$ & $284(83.3)$ & \\
\hline adenocarcinoma & $65(12.7)$ & $46(13.5)$ & \\
\hline adenosquamous carcinoma & $28(5.5)$ & $11(3.2)$ & \\
\hline FIGO stage & & & $<0.001$ \\
\hline $\mathrm{IA}$ & $24(4.7)$ & $17(5.0)$ & \\
\hline IB1 & $234(45.9)$ & $237(69.5)$ & \\
\hline |B2-IIA & $252(49.4)$ & $87(25.5)$ & \\
\hline Histologic grading & & & 0.149 \\
\hline G1 & $21(4.1)$ & $17(5.0)$ & \\
\hline G2 & $62(12.2)$ & $42(12.3)$ & \\
\hline G3 & $378(74.1)$ & $233(68.3)$ & \\
\hline Gx & $49(9.6)$ & $49(14.4)$ & \\
\hline Stromal invasion depth & & & $<0.001$ \\
\hline$<1 / 2$ & $203(39.8)$ & $170(49.9)$ & \\
\hline$\geq 1 / 2$ & $307(60.2)$ & $171(50.1)$ & \\
\hline LVSI & $308(60.4)$ & $208(61.0)$ & 0.860 \\
\hline Parametrial invasion & $63(12.4)$ & $43(12.6)$ & 0.911 \\
\hline Positive vaginal margin & $92(18.0)$ & $45(13.2)$ & 0.060 \\
\hline Lymph node metastasis & $99(19.4)$ & $73(21.4)$ & 0.477 \\
\hline Hospital stay, median (range, days) & $8(3-30)$ & $7(3-21)$ & $<0.001$ \\
\hline Duration of surgery, median (range, min) & $200(85-510)$ & $240(75-450)$ & $<0.001$ \\
\hline Estimated blood loss, median (range, ml) & $400(50-2500)$ & $200(10-4500)$ & $<0.001$ \\
\hline Postoperative adjuvant treatment & $399(78.2)$ & $285(83.6)$ & 0.054 \\
\hline
\end{tabular}

BMI, body-mass index; FIGO, International Federation of Gynecology and Obstetrics; LVSI, Iymph-vascular space invasion.

of age, menopause, BMI, histologic subtype, and grading. Advanced FIGO stage and deep cervical stromal invasion were more frequent in the open surgery group. The median length of hospital stay was eight days (range 3-30) in the open surgery group and seven days (range $3-21)$ in the MIS group $(P<0.001)$. The median duration of surgery was 200 minutes (range $85-510$ ) in the open surgery group and 240 minutes (range 75-450) in the MIS group $(P<0.001)$, and the median volume of blood loss was $400 \mathrm{~mL}$ (range, 50-2500 mL) in the open surgery group and 200 $\mathrm{mL}$ (range, $10-4500 \mathrm{~mL}$ ) in the MIS group $(P<0.001)$.

\section{Clinical Outcomes in Different Surgical Approaches}

The median follow-up duration was 88.6 and 62.2 months in the open surgery and MIS groups, respectively. With regard to the three- and five-year OS and DFS, statistically significant differences were not observed (three-year OS, 94.5\% vs. $96.4 \%$; five-year OS, 93.4\% vs. 96.0\%; $P=0.291$; three-year DFS: $94.4 \%$ vs. $94.6 \%$, five-year DFS: $92.8 \%$ vs. $94.2 \%, P=0.585)$. After adjusting for age, BMI, FIGO stage, histologic subtype, and grading, identical results were obtained using multivariable Cox proportional hazards regression models [OS: $P=0.894$, HR 1.039, 95\%CI $(0.593,1.820)$; DFS, $P=0.647$; HR, 0.891; 95\% CI $(0.544,1.460)$; Figure 1]. In addition, we analyzed the survival outcomes of the open surgery and MIS groups for every surgeon and found that the survival outcomes were still not statistically significant (all $p$-values $>0.05$ ).

\section{Survival Outcomes in Different Phases and Surgeons}

For every surgeon, we categorized patients who underwent surgery into four phases according to their sequence (phase one, 1-10 cases; phase two, 11-20 cases; phase three, 21-30 cases; phase four, more than 30 cases). Considering open surgery as an advanced technique, we did not categorize patients in this group into different phases. When stratified by surgical phases, the OS and DFS of the MIS group in phase one were significantly lower than those in later phases and in the open surgery group after adjusting for age, BMI, FIGO stage, histologic subtype, and grading. The three-year OS was $91.8 \%$ (45/49), and the fiveyear OS was $87.8 \%(43 / 49)$ in phase one of the MIS group $(P=$ 0.009 ; HR, 2.896; 95\%CI, 1.303-6.435). The three-year DFS was $91.8 \%(45 / 49)$ and the five-year DFS was $85.7 \%(42 / 49)$ in phase one of the MIS group ( $P=0.009$; HR, 2.712; 95\%CI, 1.289-5.706; Figure 2). We obtained similar results when analyzing the surgeons separately (Table 2). Statistical differences were observed in the median number of resected lymph nodes $(p<$ 0.001). There were no significant differences among the four phases with respect to perioperative characteristics, including hospital stay, duration of surgery, volume of blood loss, and volume of $\mathrm{CO}_{2}$ pneumoperitoneum after adjustment (Table 3).

When comparing different surgeons, the survival outcomes were not statistically significant after adjustment. Furthermore, the subgroup analysis in the corresponding phases showed the same result (all $P>0.05$; Figure 3 ). 

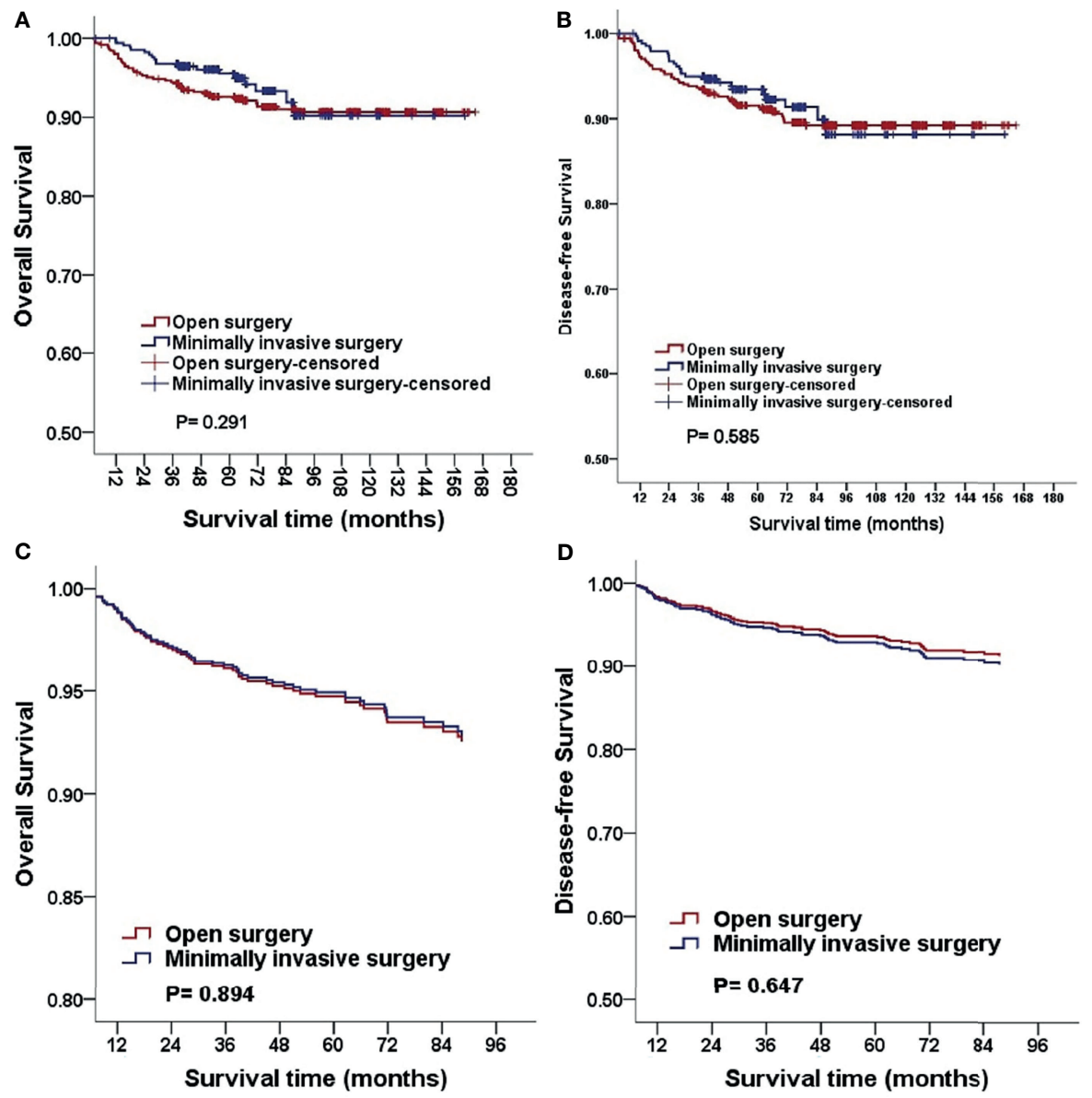

FIGURE 1 | Kaplan-Meier analysis of the OS and DFS for patients stratified by surgical approach (A, B). The OS and DFS adjusted for clinicopathological factors for patients stratified by surgical approach (C, D). MIS, minimally invasive surgery.
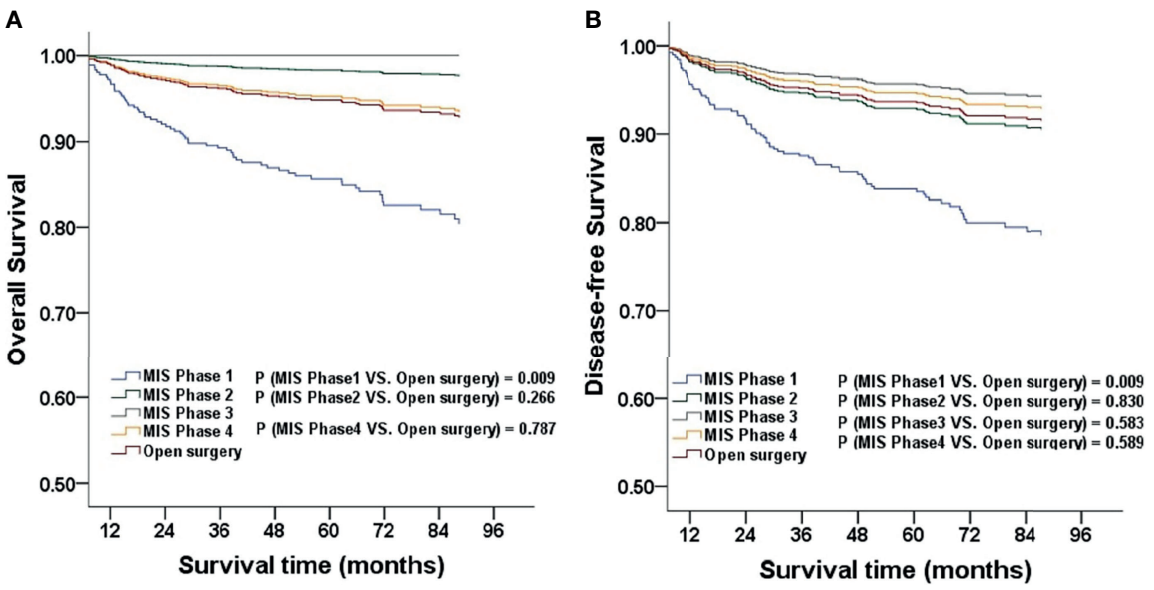

FIGURE 2 | The OS and DFS adjusted for clinicopathological factors for patients stratified by phase (A, B). MIS, minimally invasive surgery. 
TABLE 2 | Survival outcomes of different surgeons in different phases in minimally invasive surgery.

\begin{tabular}{|c|c|c|c|c|c|c|c|c|c|}
\hline \multirow[t]{2}{*}{ Variable } & & \multicolumn{4}{|c|}{ Overall survival } & \multicolumn{4}{|c|}{ Disease-free survival } \\
\hline & & 3-year (\%) & 5-year (\%) & HR $(95 \% \mathrm{Cl})$ & $P$ value & 3-year (\%) & 5-year (\%) & HR $(95 \% \mathrm{Cl})$ & $P$ value \\
\hline \multicolumn{2}{|l|}{ Open surgery } & 94.3 & 92.4 & 1 (ref.) & - & 93.4 & 91.3 & 1 (ref.) & - \\
\hline \multirow[t]{4}{*}{ Surgeon A } & P1 & 100 & 90 & $1.834(0.237-14.17)$ & 0.561 & 90 & 80 & $3.464(0.792-15.162)$ & 0.099 \\
\hline & P2 & 100 & 100 & - & - & 100 & 100 & - & - \\
\hline & P3 & 100 & 100 & - & - & 100 & 100 & - & - \\
\hline & P4 & 100 & 100 & - & - & 100 & 100 & - & - \\
\hline \multirow[t]{4}{*}{ Surgeon B } & P1 & 100 & 100 & - & - & 100 & 100 & - & - \\
\hline & P2 & 100 & 100 & - & - & 100 & 90 & 1.567 (0.199-12.341) & 0.670 \\
\hline & P3 & 100 & 100 & - & - & 90 & 90 & 2.627 (0.336-20.542) & 0.357 \\
\hline & P4 & 100 & 100 & - & - & 100 & 100 & - & - \\
\hline \multirow[t]{4}{*}{ Surgeon C } & P1 & 90 & 80 & 2.595 (0.732-9.197) & 0.140 & 90 & 80 & 2.397 (0.698-8.226) & 0.165 \\
\hline & P2 & 100 & 100 & - & - & 88.9 & 88.9 & $1.368(0.183-10.231)$ & 0.760 \\
\hline & P3 & 100 & 100 & - & - & 88.9 & 88.9 & $1.501(0.202-11.126)$ & 0.691 \\
\hline & P4 & 97.9 & 93.4 & $0.742(0.263-2.094)$ & 0.573 & 96.8 & 93.7 & 0.617 (0.220-1.725) & 0.357 \\
\hline \multirow[t]{4}{*}{ Surgeon D } & P1 & 77.8 & 77.8 & $5.866(1.301-26.441)$ & 0.021 & 77.8 & 77.8 & 4.696 (1.061-20.776) & 0.042 \\
\hline & P2 & 100 & 90 & $1.273(0.164-9.901)$ & 0.817 & 90 & 90 & $1.261(0.165-9.662)$ & 0.823 \\
\hline & P3 & 100 & 100 & - & - & 100 & 100 & - & - \\
\hline & P4 & 91.8 & 91.8 & $1.420(0.585-3.446)$ & 0.439 & 91.7 & 89.3 & 1.407 (0.621-3.187) & 0.414 \\
\hline \multirow[t]{4}{*}{ Surgeon E } & P1 & 90 & 78.8 & $5.020(1.106-22.790)$ & 0.037 & 90 & 78.8 & $4.256(0.951-19.037)$ & 0.058 \\
\hline & P2 & 100 & 100 & - & - & 100 & 90 & $1.866(0.247-14.102)$ & 0.546 \\
\hline & P3 & 100 & 100 & - & - & 100 & 100 & - & - \\
\hline & P4 & 100 & 100 & - & - & 100 & 100 & - & - \\
\hline \multirow[t]{4}{*}{ Total } & P1 & 91.8 & 87.8 & 2.896 (1.303-6.435) & 0.009 & 91.8 & 85.7 & 2.712 (1.289-5.706) & 0.009 \\
\hline & P2 & 100 & 96.7 & $0.321(0.043-2.381)$ & 0.266 & 93.9 & 91.6 & 1.122 (0.392-3.213) & 0.830 \\
\hline & P3 & 100 & 100 & - & - & 94.9 & 94.9 & $0.671(0.162-2.782)$ & 0.583 \\
\hline & P4 & 96 & 92.1 & 0.907 (0.446-1.843) & 0.787 & 95.5 & 91.6 & $0.831(0.426-1.624)$ & 0.589 \\
\hline
\end{tabular}

$P$ values $<0.05$ are in bold. $P$, phase; $H R$, hazard ratio; $C l$, confidence interval; ref, reference.

\section{DISCUSSION}

The World Health Organization proposed that patients with cervical cancer must be managed appropriately to accelerate the elimination of the disease, prompting more attention to the treatment of cervical cancer in recent years. Previous studies demonstrated that MIS and open surgery had comparable oncological outcomes (14-16). In contrast, several recent studies showed poorer oncological outcomes, especially lower DFS in patients treated with MIS $(3,17-19)$. As the findings were conflicting, the superiority or inferiority of MIS remains unclear. In our study, there were no statistical differences in the MIS and open surgery groups with regard to three- and five-year OS and DFS, and the subgroup analysis performed by different surgeons reached the same conclusion. However, when we compared different phases in MIS with open surgery, the OS and DFS of phase one were in line with those of the LACC trial. The perioperative outcomes such as hospital stay and blood loss were significantly better in the MIS group, whereas the duration of surgery was significantly shorter in the open surgery group, which is in accordance with many other studies.

According to the NCCN guideline (2), the standard approach for $\mathrm{RH}$ is the open surgery, and the oncologic risks of MIS should be informed carefully to patients given the findings of poorer survival outcomes of MIS recently. The development of MIS was affected by relevant research works. Therefore, it is essential to clear the reasonable application of MIS to achieve maximum benefits for patients. Pedestrian et al. showed that patients with tumor dimension less than two centimeters still suitable for MIS, but more studies are needed to refine on criteria (20).

Several potential reasons may explain the limitations of MIS, such as the use of a uterine manipulator, insufflation gas, intracorporeal colpotomy under $\mathrm{CO}_{2}$ pneumoperitoneum, and the proficiency of the surgeon (7, 21-23). Gynecologic

TABLE 3 | Perioperative outcomes in different phases in minimally invasive surgery.

\begin{tabular}{|c|c|c|c|c|c|}
\hline \multirow[t]{2}{*}{ Characteristic } & \multicolumn{4}{|c|}{ Minimally invasive surgery } & \multirow[b]{2}{*}{$P$ value } \\
\hline & Phase 1 & Phase 2 & Phase 3 & Phase 4 & \\
\hline Hospital stay (days) (mean \pm SD) & $7.88 \pm 2.88$ & $7.32 \pm 2.33$ & $7.50 \pm 2.39$ & $7.15 \pm 2.14$ & 0.235 \\
\hline Duration of surgery (min) (mean $\pm \mathrm{SD}$ ) & $252.73 \pm 50.85$ & $25.72 \pm 56.88$ & $238.89 \pm 45.39$ & $245.37 \pm 62.08$ & 0.659 \\
\hline Estimated blood loss, median (range, ml) & $175(20-800)$ & $200(40-600)$ & $200(80-4500)$ & $200(10-1000)$ & 0.479 \\
\hline CO2 pneumoperitoneum, median (range, L) & $590(80-1200)$ & $483.5(160-1090)$ & $669(255-1200)$ & $684(100-1300)$ & 0.123 \\
\hline No. of lymph nodes resected, median (range) & $18.5(4-33)$ & $19(3-57)$ & $17.5(6-50)$ & $23(2-53)$ & $<0.001$ \\
\hline
\end{tabular}

$S D$, standard deviation. 

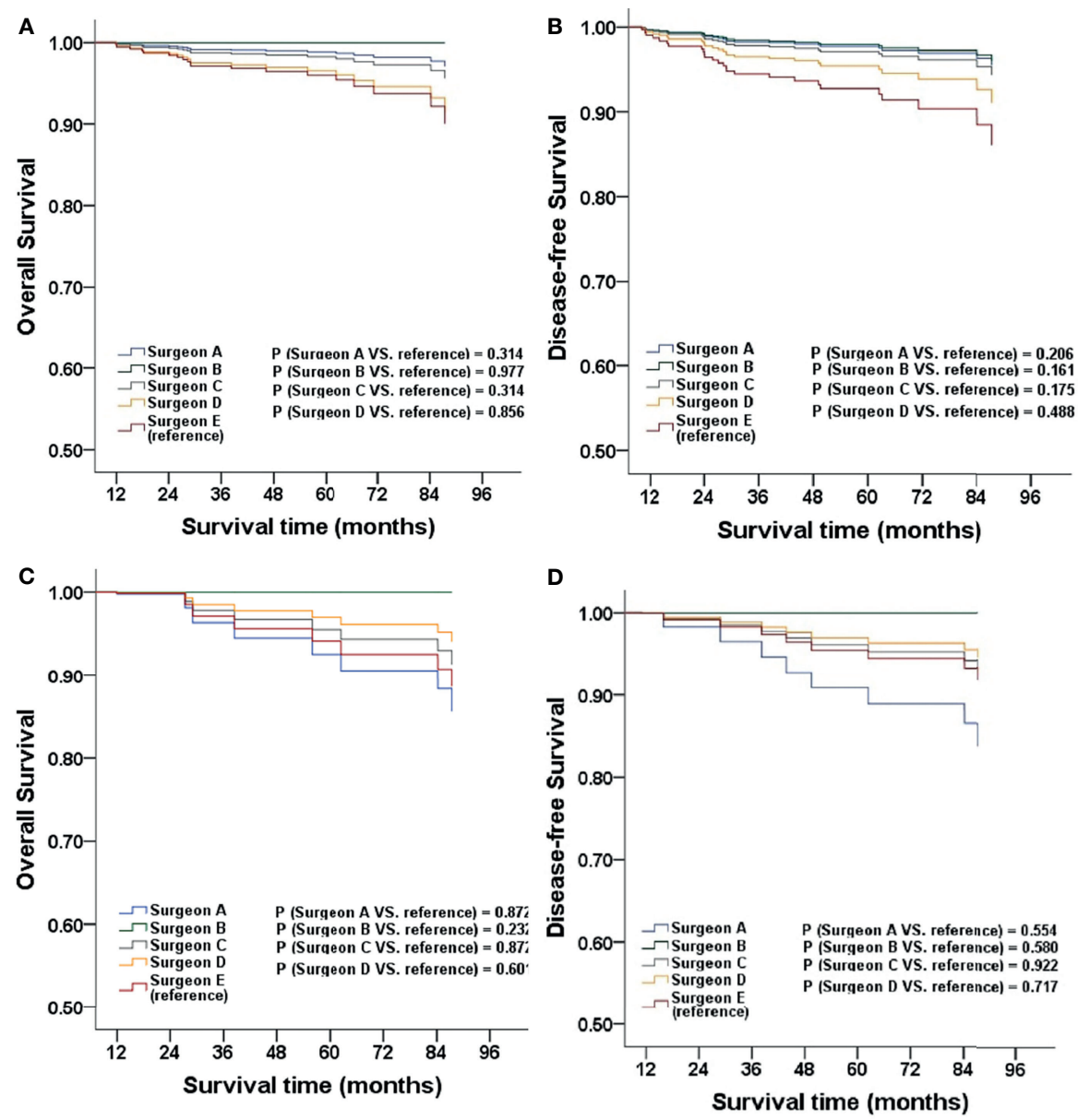

FIGURE 3 | The OS and DFS adjusted for clinicopathological factors for patients stratified by surgeon (A, B), patients stratified by surgeon in MIS phase one (C, D)

oncologists have designed innovative techniques and devices to reduce the potential negative consequences of these risks in recent years. For example, Hiroyuki Kanao et al. devised the "no-look, no-touch" technique for preventing intraoperative tumor spillage (24). Moreover, Peng Yuan et al. performed abdominal uterine manipulation and enclosed colpotomy (23). However, prospective studies are needed to evaluate the effects of these surgical techniques on oncological outcomes.

Previous studies have examined the learning curve in terms of some clinical parameters, such as the number of cases needed to reach a stable operation duration or to obtain a relatively low hemorrhage volume (25). Survival outcomes have rarely been reported. A recent study by Kim et al. concluded that improvement of surgical performance could be achieved after 13 cases of MIS (9). Pedestrian et al. demonstrated that the peak of reduction of the recurrence risk was the $19^{\text {th }}$ MIS (26). Sert et al. showed that there was a higher number of recurrences in the first 50 cases (27). In this regard, our findings were in line with the results of previous studies that the first ten cases were significantly inferior to subsequent cases. Moreover, favorable survival outcomes observed after the first ten cases also demonstrated the effect of surgeon proficiency. There was no statistical significance when comparing the different surgeons, indicating that favorable outcomes can be obtained by practicing the MIS technique after a certain number of cases. Based on our findings, we offer the following suggestions. First, surgeons should undergo standardized training to improve their surgical skills (28). Second, surgeons performing MIS should have some standard qualification. For example, surgeons can only perform laparoscopic surgery after completing a certain number of virtual surgeries through surgery simulators. Third, beginners should be overseen by experienced surgeons, particularly for the first dozen or so cases.

The main strength of this study is the large sample size. In addition, compared with other studies, we analyzed more clinical parameters, making our results more robust and applicable to a larger population. However, our study has several limitations. Due to its retrospective nature, there could be bias in the patient selection. Furthermore, we did not evaluate the effect of different surgical techniques and devices in different phases. More studies on specific surgical approaches and techniques, including conventional multiport laparoscopic and laparo-endoscopic 
single-site surgery with/without technological innovations, are needed. Although there was no robotic case in our study, some relevant studies showed that robotic and laparoscopic approaches were similar in perioperative and postoperative outcomes (29), future studies could consider this aspect.

In conclusion, our retrospective study demonstrated that phase one cases of MIS had lower OS and DFS than those in later phases or that underwent open surgery. Thus, we suggest that the proficiency of the operating surgeon is associated with the survival outcomes of MIS. More favorable outcomes can be obtained after a certain number of MIS cases have been performed.

\section{DATA AVAILABILITY STATEMENT}

The original contributions presented in the study are included in the article/supplementary material. Further inquiries can be directed to the corresponding author.

\section{ETHICS STATEMENT}

The studies involving human participants were reviewed and approved by the Institutional Ethics Committee of West China

\section{REFERENCES}

1. World Health Organization. WHO Guideline for Screening and Treatment of Cervical Precancer Lesions for Cervical Cancer Prevention (2021). Available at: https://wwwwhoint/publications/i/item/9789240030824.

2. Koh WJ, Abu-Rustum NR, Bean S, Bradley K, Campos SM, Cho KR, et al. Cervical Cancer, Version 3.2019, NCCN Clinical Practice Guidelines in Oncology. J Natl Compr Canc Netw (2019) 17:64-84. doi: 10.6004/jnccn.2019.0001

3. Ramirez PT, Frumovitz M, Pareja R, Lopez A, Vieira M, Ribeiro R, et al. Minimally Invasive Versus Abdominal Radical Hysterectomy for Cervical Cancer. N Engl J Med (2018) 379:1895-904. doi: 10.1056/NEJMoa1806395

4. Mowat A, Maher C, Ballard E. Surgical Outcomes for Low-Volume vs HighVolume Surgeons in Gynecology Surgery: A Systematic Review and MetaAnalysis. Am J Obstet Gynecol (2016) 215:21-33. doi: 10.1016/j.ajog.2016. 02.048

5. Nezhat FR, Ananth CV, Vintzileos AM. The Two Achilles Heels of Surgical Randomized Controlled Trials: Differences in Surgical Skills and Reporting of Average Performance. Am J Obstet Gynecol (2019) 221:230-2. doi: 10.1016/ j.ajog.2019.05.017

6. Casarin J, Buda A, Bogani G, Fanfani F, Papadia A, Ceccaroni M, et al. Predictors of Recurrence Following Laparoscopic Radical Hysterectomy for Early-Stage Cervical Cancer: A Multi-Institutional Study. Gynecol Oncol (2020) 159:164-70. doi: 10.1016/j.ygyno.2020.06.508

7. Chen S, Zheng Y, Tong L, Zhao X, Chen L, Wang Y. Laparoendoscopic Single-Site Radical Hysterectomy With Vaginal Closure and Without Uterine Manipulator for FIGO IB1 Cervical Cancer. J Minim Invasive Gynecol (2020) 27:1471-2. doi: 10.1016/j.jmig.2020.01.003

8. Li LY, Wen LY, Park SH, Nam EJ, Lee JY, Kim S, et al. Impact of the Learning Curve on the Survival of Abdominal or Minimally Invasive Radical Hysterectomy for Early-Stage Cervical Cancer. Cancer Res Treat (2021) 53:243-51. doi: 10.4143/crt.2020.063

9. Kim S, Min KJ, Lee S, Hong JH, Song JY, Lee JK, et al. Learning Curve Could Affect Oncologic Outcome of Minimally Invasive Radical Hysterectomy for Cervical Cancer. Asian J Surg (2021) 44:174-80. doi: 10.1016/ j.asjsur.2020.05.006

10. Liu Y, Li L, Wu M, Ma S, Tan X, Zhong S, et al. The Impact of the Surgical Routes and Learning Curve of Radical Hysterectomy on the Survival
Second University Hospital. The patients/participants provided their written informed consent to participate in this study.

\section{AUTHOR CONTRIBUTIONS}

YY and ZYL contributed to data collection and study design. YY and $\mathrm{YH}$ contributed to data analysis. All authors contributed to the article and approved the submitted version.

\section{FUNDING}

This study was supported by grants from the Sichuan Youth Foundation of Science of Technology (Grant number: 2015JQ0026).

\section{ACKNOWLEDGMENTS}

The authors are grateful to the Department of Gynecology and Obstetrics, West China Second University Hospital, for supplying the data and all the patients.

Outcomes in Stage IB Cervical Cancer: A Retrospective Cohort Study. Int J Surg (2019) 68:72-7. doi: 10.1016/j.ijsu.2019.06.009

11. Querleu D, Morrow CP. Classification of Radical Hysterectomy. Lancet Oncol (2008) 9:297-303. doi: 10.1016/s1470-2045(08)70074-3

12. Cibula D, Abu-Rustum NR, Benedetti-Panici P, Köhler C, Raspagliesi F, Querleu D, et al. New Classification System of Radical Hysterectomy: Emphasis on a Three-Dimensional Anatomic Template for Parametrial Resection. Gynecol Oncol (2011) 122:264-8. doi: 10.1016/j.ygyno.2011.04.029

13. Austin PC, White IR, Lee DS, van Buuren S. Missing Data in Clinical Research: A Tutorial on Multiple Imputation. Can J Cardiol (2020) 37:13227-31. doi: 10.1016/j.cjca.2020.11.010

14. Shah CA, Beck T, Liao JB, Giannakopoulos NV, Veljovich D, Paley P. Surgical and Oncologic Outcomes After Robotic Radical Hysterectomy as Compared to Open Radical Hysterectomy in the Treatment of Early Cervical Cancer. J Gynecol Oncol (2017) 28:e82. doi: 10.3802/jgo.2017.28.e82

15. Wallin E, Floter Radestad A, Falconer H. Introduction of Robot-Assisted Radical Hysterectomy for Early Stage Cervical Cancer: Impact on Complications, Costs and Oncologic Outcome. Acta Obstet Gynecol Scand (2017) 96:536-42. doi: 10.1111/aogs.13112

16. Mendivil AA, Rettenmaier MA, Abaid LN, Brown JV3rd, Micha JP, Lopez KL, et al. Survival Rate Comparisons Amongst Cervical Cancer Patients Treated With an Open, Robotic-Assisted or Laparoscopic Radical Hysterectomy: A Five Year Experience. Surg Oncol (2016) 25:66-71. doi: 10.1016/j.suronc. 2015.09.004

17. Uppal S, Gehrig PA, Peng K, Bixel KL, Matsuo K, Vetter MH, et al. Recurrence Rates in Patients With Cervical Cancer Treated With Abdominal Versus Minimally Invasive Radical Hysterectomy: A Multi-Institutional Retrospective Review Study. J Clin Oncol (2020) 38:1030-40. doi: 10.1200/jco.19.03012

18. Chao X, Li L, Wu M, Ma S, Tan X, Zhong S, et al. Efficacy of Different Surgical Approaches in the Clinical and Survival Outcomes of Patients With EarlyStage Cervical Cancer: Protocol of a Phase III Multicentre Randomised Controlled Trial in China. BMJ Open (2019) 9:e029055. doi: 10.1136/ bmjopen-2019-029055

19. Melamed A, Margul DJ, Chen L, Keating NL, Del Carmen MG, Yang J, et al. Survival After Minimally Invasive Radical Hysterectomy for Early-Stage Cervical Cancer. N Engl J Med (2018) 379:1905-14. doi: 10.1056/ NEJMoa1804923 
20. Pedestrian Anchora L, Turco LC, Bizzarri N, Capozzi VA, Lombisani A, Chiantera V, et al. How to Select Early-Stage Cervical Cancer Patients Still Suitable for Laparoscopic Radical Hysterectomy: A Propensity-Matched Study. Ann Surg Oncol (2020) 27(6):1947-55. doi: 10.1245/s10434-019-08162-5

21. Klapdor R, Hertel H, Hillemanns P, Röttger M, Soergel P, Kuehnle E, et al. Peritoneal Contamination With ICG-Stained Cervical Secretion as Surrogate for Potential Cervical Cancer Tumor Cell Dissemination: A Proof-of-Principle Study for Laparoscopic Hysterectomy. Acta Obstet Gynecol Scand (2019) 98:1398-403. doi: 10.1111/aogs.13681

22. Kampers J, Gerhardt E, Sibbertsen P, Flock T, Klapdor R, Hertel H, et al. Protective Operative Techniques in Radical Hysterectomy in Early Cervical Carcinoma and Their Influence on Disease-Free and Overall Survival: A Systematic Review and Meta-Analysis of Risk Groups. Arch Gynecol Obstet (2021) 304:577-87. doi: 10.1007/s00404-021-06082-y

23. Yuan P, Liu Z, Qi J, Yang X, Hu T, Tan H. Laparoscopic Radical Hysterectomy With Enclosed Colpotomy and Without the Use of Uterine Manipulator for Early-Stage Cervical Cancer. J Minim Invasive Gynecol (2019) 26:1193-8. doi: 10.1016/j.jmig.2019.01.016

24. Kanao H, Matsuo K, Aoki Y, Tanigawa T, Nomura H, Okamoto S, et al. Feasibility and Outcome of Total Laparoscopic Radical Hysterectomy With No-Look No-Touch Technique for FIGO IB1cervical Cancer. J Gynecol Oncol (2019) 30:e71. doi: 10.3802/jgo.2019.30.e71

25. Heo YJ, Kim S, Min KJ, Lee S, Hong JH, Lee JK, et al. The Comparison of Surgical Outcomes and Learning Curves of Radical Hysterectomy by Laparoscopy and Robotic System for Cervical Cancer: An Experience of a Single Surgeon. Obstet Gynecol Sci (2018) 61:468-76. doi: 10.5468/ogs.2018.61.4.468

26. Pedestrian Anchora L, Bizzarri N, Gallotta V, Chiantera V, Fanfani F, Fagotti A, et al. Impact of Surgeon Learning Curve in Minimally Invasive Radical Hysterectomy on Early Stage Cervical Cancer Patient Survival. Facts Views Vis Obgyn (2021) 13(3):233-44. doi: 10.52054/FVVO.13.3.035
27. Hoogendam JP, Verheijen RH, Wegner I, Zweemer RP. Oncological Outcome and Long-Term Complications in Robot-Assisted Radical Surgery for Early Stage Cervical Cancer: An Observational Cohort Study. Bjog (2014) 121:153845. doi: 10.1111/1471-0528.12822

28. Moulton CA, Dubrowski A, Macrae H, Graham B, Grober E, Reznick R. Teaching Surgical Skills: What Kind of Practice Makes Perfect?: A Randomized, Controlled Trial. Ann Surg (2006) 244:400-9. doi: 10.1097/ 01.sla.0000234808.85789.6a

29. Gallotta V, Conte C, Federico A, Vizzielli G, Gueli Alletti S, Tortorella L, et al. Robotic Versus Laparoscopic Radical Hysterectomy in Early Cervical Cancer: A Case Matched Control Study. Eur J Surg Oncol (2018) 44(6):754-9. doi: 10.1016/j.ejso.2018.01.092

Conflict of Interest: The authors declare that the research was conducted in the absence of any commercial or financial relationships that could be construed as a potential conflict of interest.

Publisher's Note: All claims expressed in this article are solely those of the authors and do not necessarily represent those of their affiliated organizations, or those of the publisher, the editors and the reviewers. Any product that may be evaluated in this article, or claim that may be made by its manufacturer, is not guaranteed or endorsed by the publisher.

Copyright (C) 2021 Yang, Huang and Li. This is an open-access article distributed under the terms of the Creative Commons Attribution License (CC BY). The use, distribution or reproduction in other forums is permitted, provided the original author(s) and the copyright owner(s) are credited and that the original publication in this journal is cited, in accordance with accepted academic practice. No use, distribution or reproduction is permitted which does not comply with these terms. 